\title{
Time-dependent Picosecond Transient Absorption Spectra of 9-Acetylanthracene, Benzophenone and Acridine in Solution
}

\author{
KUMAO HAMANOUE, KAZUO NAKAJIMA, TOSHIHARU HIDAKA, \\ TOSHIHIRO NAKAYAMA and HIROSHI TERANISHI
}

Department of Chemistry, Faculty of Technology, Kyoto Institute of Technology, Matsugasaki, Sakyo-ku, Kyoto 606, Japan

(Received June 22, 1983)

The transient absorption spectra of the title compounds in solutions at room temperature have been measured on the picosecond time scale. For 9-acetylanthracene and acridine there were measurable changes of the spectral shapes in the first picosecond region, while no spectral change was observed for benzophenone.

\section{INTRODUCTION}

Recently, Hochstrasser et al. ${ }^{1}$ reported on the time-dependent picosecond transient absorption spectra of a number of compounds in solution at room temperature. All the spectra, at different delay times, were normalized to the same peak height to facilitate comparison of the band shapes. The spectra obtained by this procedure suggest that vibrationally unrelaxed molecules are present in the solutions for times up to tens of picoseconds after optical excitation. In some cases new information was obtained about the electronic states whose spectra had been unobserved by that time. One should note, however, that such a normalization procedure for comparison 
of the band shapes is liable to amplify errors in weak absorption bands by the multiplication of small errors, such as baseline drifts.

In the present study, we observed the transient absorptions of 9-acetylanthracene, benzophenone and acridine at a given delay time by changing the sample concentration or the intensity of excitation light, and weak spectra were normalized with reference to the corresponding strong ones. Taking into account the errors caused by the normalization, we observed the transient absorptions at different delay times to see whether the essential spectral narrowing and shift were present in the first picosecond region.

\section{EXPERIMENTAL}

9-Acetylanthracene was synthesized by the method of Hawkins. ${ }^{2}$ G.R.-grade benzophenone (Wako) was recrystallized three times from ligroin. Zone-refined acridine (Tokyo Kasei) was used without further purification. The solvents (acetonitrile, $n$-heptane, $n$-hexane) were of spectral grade (Dojin) and were used without further purification. Sample solutions in a cell of $2 \mathrm{~mm}$ pathlength were not deaerated. The transient absorption spectra were measured at room temperature.

The details of our picosecond transient absorption spectrometer have been given elsewhere. ${ }^{3}$ The second harmonic $(347.2 \mathrm{~nm})$ from a mode-locked ruby laser was used to excite the sample. The mean pulse width $(26 \mathrm{ps})$ and the time-zero point, $t=0$, were determined from the overlap of excitation and probe pulses by measuring the buildup of $T_{n} \leftarrow T_{1}$ absorption of benzophenone (in $n$-heptane) at $530 \mathrm{~nm}$. A double-beam optical arrangement was adopted, and absorption spectra in the $200 \mathrm{~nm}$ scanning region were measured with two multichannel photodiode systems. The three most probable spectra were averaged.

\section{NORMALIZATION OF SPECTRA}

The reproducibility and stability of a mode-locked ruby laser were not stated to have been well controlled. Since our absorption spectrum was obtained by two laser shots, small errors such as baseline drifts were inevitable in the calculation of a double-beam absorption spectrum, i.e., the channel-by-channel subtraction. 
Thus, the following two procedures were carried out in the normalization of the weak absorption spectrum (Spectrum B) to a strong one (Spectrum A) due to the same species.

(1) Normalization I. Let the true absorbances of Spectra A and B be $I_{A}(\lambda)$ and $I_{B}(\lambda)$ with systematic drifts of $\Delta_{A}$ and $\Delta_{B}$ in the baseline, respectively, where $\lambda$ is the wavelength for a given absorption. When the band maximum of Spectrum B is normalized to that of Spectrum A at $\lambda=\lambda_{1}$, one obtains

$$
\alpha\left[I_{B}(\lambda)+\Delta_{B}\right]=I_{A}(\lambda)+\Delta_{A}+\left(\alpha \Delta_{B}-\Delta_{A}\right)\left[1-\frac{I_{A}(\lambda)}{I_{A}\left(\lambda_{1}\right)}\right]
$$

where $\alpha$ is the normalization factor, i.e.,

$$
\alpha=\left[I_{A}\left(\lambda_{1}\right)+\Delta_{A}\right] /\left[I_{B}\left(\lambda_{1}\right)+\Delta_{B}\right]
$$

Since each baseline is nearly horizontal, and $\Delta_{A}$ and $\Delta_{B}$ are less than \pm 0.02 absorbance units for our system, Eq. (1) gives two normalized spectra depending on the positive and negative values of $\left(\alpha \Delta_{B}-\right.$ $\Delta_{A}$ ), i.e., Spectra $\mathrm{C}$ and $\mathrm{C}^{\prime}$ in Figure 1-I.

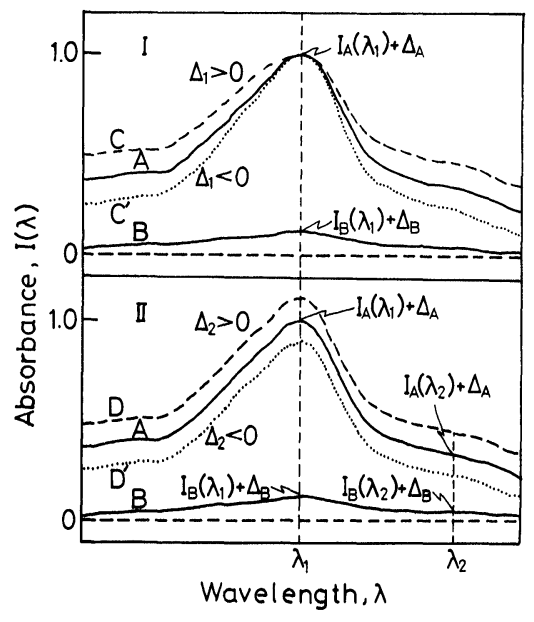

FIGURE 1 Normalization of a weak absorption (B) with reference to that of a strong one (A). (C) or $\left(C^{\prime}\right)$, normalized by Normalization I; (D) or $\left(D^{\prime}\right)$, normalized by Normalization II; $\Delta_{1}=\alpha \Delta_{B}-\Delta_{A} ; \Delta_{2}=\beta \Delta_{B}-\Delta_{A}$. 
(2) Normalization II. Let a normalization factor $\beta$ be defined as follows:

$$
\beta=\frac{\left[I_{A}\left(\lambda_{1}\right)+\Delta_{A}\right]-\left[I_{A}\left(\lambda_{2}\right)+\Delta_{A}\right]}{\left[I_{B}\left(\lambda_{1}\right)+\Delta_{B}\right]-\left[I_{B}\left(\lambda_{2}\right)+\Delta_{B}\right]}=\frac{I_{A}\left(\lambda_{1}\right)-I_{A}\left(\lambda_{2}\right)}{I_{B}\left(\lambda_{1}\right)-I_{B}\left(\lambda_{2}\right)}
$$

where $\lambda_{2}$ denotes an appropriate wavelength other than $\lambda_{1}$ at the band maximum. Since $I_{A}(\lambda)$ and $I_{B}(\lambda)$ represent the true absorbances of Spectra A and B, respectively, the intensity ratio $I_{A}(\lambda) / I_{B}(\lambda)$ should be independent of $\lambda$, so that $I_{A}(\lambda) / I_{B}(\lambda)=I_{A}\left(\lambda_{1}\right) / I_{B}\left(\lambda_{1}\right)=$ $I_{A}\left(\lambda_{2}\right) / I_{B}\left(\lambda_{2}\right)$. It then follows from Eq. (2) that $\beta=I_{A}(\lambda) / I_{B}(\lambda)$. Therefore, one obtains

$$
\beta\left[I_{B}(\lambda)+\Delta_{B}\right]=I_{A}(\lambda)+\Delta_{A}+\left(\beta \Delta_{B}-\Delta_{A}\right)
$$

Eq. (3) gives two normalized spectra as illustrated in Figure 1-II (Spectra $\mathrm{D}$ and $\mathrm{D}^{\prime}$ ), depending on the positive and negative values of $\left(\beta \Delta_{B}-\Delta_{A}\right)$. The basic difference between the two types of normalizations is that Normalization I always gives spurious absorptions at $\lambda \neq \lambda_{1}$, especially in the region of very weak absorption, while Normalization II gives Spectrum A by a vertical shift of $\left(\beta \Delta_{B}-\Delta_{A}\right)$ in absorbance units after multiplication of Spectrum B by $\beta$. Namely, in the case where strong and weak absorptions are thought to belong to the same species, Normalization II should, in principle, provide smaller errors than Normalization I.

\section{RESULTS AND DISCUSSION}

Figures 2-I and II show the transient absorptions of 9-acetylanthracene $\left(2.0 \times 10^{-4} \mathrm{M}\right)$ in acetonitrile at a delay time of $320 \mathrm{ps}$. The lowest trace in Figure 2-I shows a typical three-cycle baseline spectrum. The systematic deviation from zero is everywhere less than 0.02 absorbance units. Spectra A and B (solid lines), taken with the usual $\left(I_{e}\right)$ and attenuated $\left(0.15 \times I_{e}\right)$ excitation-light intensities, respectively, can be assigned to the $T_{n} \leftarrow T_{1}$ absorption of vibrationally relaxed 9-acetylanthracene. ${ }^{4}$

When the band maximum of Spectrum B is normalized to that of Spectrum A at $423 \mathrm{~nm}$ by Normalization I, Spectrum C (dotted line) is obtained. On the other hand, Spectrum $C^{\prime}$ (dotted line) is obtained by Normalization II, in which case the normalization factor $\beta$ is 


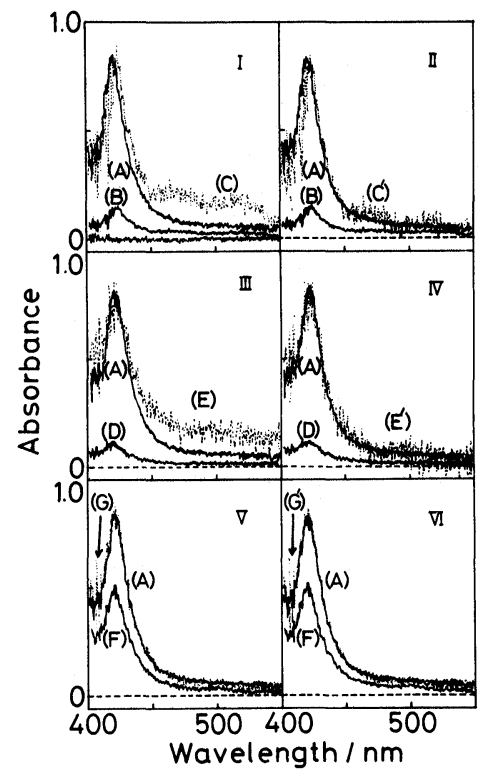

FIGURE 2 Transient absorptions of 9-acetylanthracene in acetonitrile at a delay time of 320 ps. (A), by a usual condition; (B), by attenuating the excitation-light intensity to $15 \%$ of $(\mathrm{A}) ;(\mathrm{D})$ and $(\mathrm{F})$, by reducing the sample concentration to $1 / 10$ and $1 / 2$ of $(A)$, respectively; (C), (E) and (G), normalized at $423 \mathrm{~nm}$ by Normalization I; $\left(C^{\prime}\right),\left(E^{\prime}\right)$ and $\left(G^{\prime}\right)$, normalized at 423 and $500 \mathrm{~nm}$ by normalization II. The lowest solid line in (I) is the experimental baseline spectrum taken with no excitation.

calculated using the absorbances at 423 and $500 \mathrm{~nm}$ of Spectra A and B. Spectrum $C$ gives errors resulting in weak spurious absorptions above $450 \mathrm{~nm}$ and a spectral shift at the band maximum, whereas these errors are much smaller in Spectrum $C^{\prime}$.

Nearly the same results are obtained in Figures 2-III and IV, where a weak absorption $(D)$ was taken with a sample diluted to one-tenth of the original concentration instead of attenuating the excitation-light intensity. However, Normalization I caused no significant error when the sample concentration was one-half the original one (Figures $2-\mathrm{V}$, VI). This implies that the error caused by Normalization I is inessential if the weak absorption has a sufficient intensity.

In Figure 3, we display the transient absorptions of 9-acetylanthracene taken at various delays. The spectrum taken at $-30 \mathrm{ps}$ and 


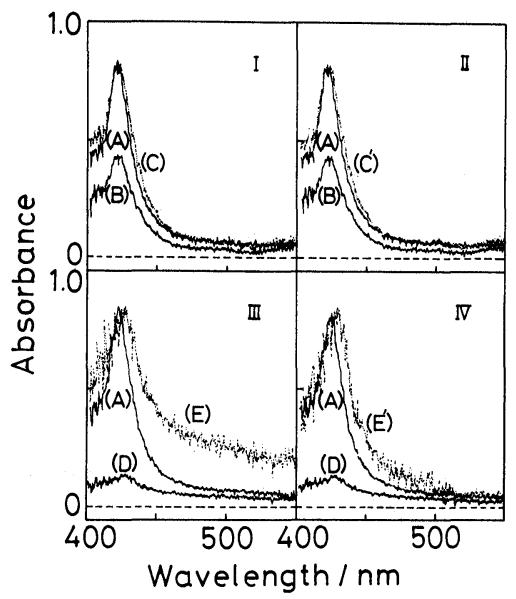

FIGURE 3 Transient absorptions of 9-acetylanthracene in acetonitrile taken at various delays. (A), (B) and (D), at $320,-10$ and -30 ps, respectively; (C) and (E), normalized by Normalization I; $\left(\mathrm{C}^{\prime}\right)$ and $\left(\mathrm{E}^{\prime}\right)$, normalized by Normalization II.

processes by Normalization II shows the essential spectral broadening and shift in comparison with that at $320 \mathrm{ps}$. Since the spectral change for 9-acetylanthracene is very similar to that for benzophenone observed by Hochstrasser et al., ${ }^{1}$ this change might be due to vibrationally unrelaxed 9-acetylanthracene.

Figures 4-I and II show the results obtained with benzophenone $\left(1.0 \times 10^{-2} \mathrm{M}\right)$ in $n$-heptane at a delay time of $320 \mathrm{ps}$. Spectra $\mathrm{A}$ and B (solid lines) were taken with the usual $\left(I_{e}\right)$ and attenuated $\left(0.1 \times I_{e}\right)$ excitation-light intensities, respectively. Since Spectrum A is very similar to the $T_{n} \leftarrow T_{1}$ absorptions of benzophenone at longer times, ${ }^{1,6-8}$ we conclude that the absorption with $\lambda_{\max }=530 \mathrm{~nm}$ is due to the $T_{n} \leftarrow T_{1}$ absorption of vibrationally relaxed benzophenone. Spectra $C$ and $C^{\prime}$ obtained by Normalization I and II are essentially identical. Nearly the same results were obtained in the normalization of the weak absorption taken by reducing the sample concentration to one-tenth.

Hochstrasser et al. ${ }^{1}$ have observed that the transient absorption of benzophenone shows spectral narrowing and shift over the first $50 \mathrm{ps}$. However, our normalized spectra taken at -20 ps (Spectra $E$ and $E^{\prime}$ in Figures 4-III and IV) show no essential spectral broadening and 


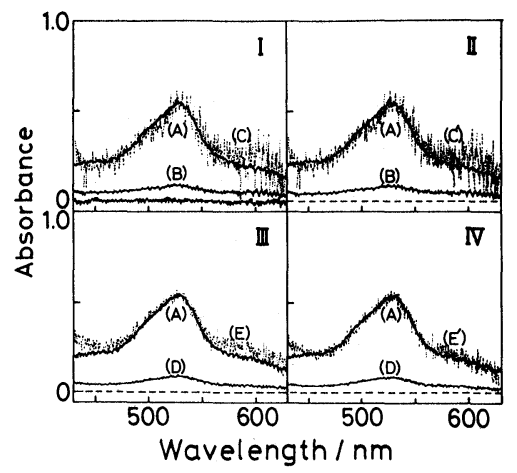

FIGURE 4 Transient absorptions of benzophenone in $n$-heptane at various delays. (A) and (B) at 320 and (D) at -20 ps, respectively. (B), by attenuating the excitationlight intensity to $10 \%$ of $(A)$; (C) and (E), normalized at $530 \mathrm{~nm}$ by Normalization I; $\left(\mathrm{C}^{\prime}\right)$ and $\left(\mathrm{E}^{\prime}\right)$, normalized at 530 and $600 \mathrm{~nm}$ by Normalization II. The lowest solid line in (I) is the experimental baseline spectrum taken with no excitation.

shift in comparison with that at 320 ps. Therefore, Spectra $E$ and $E^{\prime}$ do not appear to be vibrationally unrelaxed benzophenone.

Figures 5-I and II show the spectra of acridine $\left(2.6 \times 10^{-4} \mathrm{M}\right)$ in $n$-hexane at 320 ps delay. Spectra A and B (solid lines) were again taken with the usual $\left(I_{e}\right)$ and attenuated $\left(0.15 \times I_{e}\right)$ excitation-light

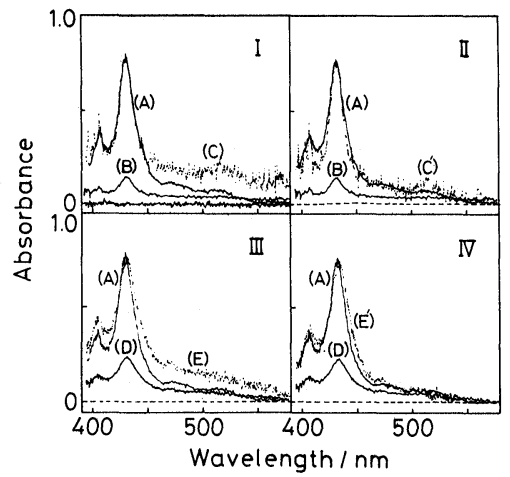

FIGURE 5 Transient absorptions of acridine in $n$-hexane at various delays. (A) and (B) at 320 and (D) at $-10 \mathrm{ps,}$, respectively. (B), by attenuating the excitation-light intensity to $15 \%$ of $(A)$; $(C)$ and $(E)$, normalized at $433 \mathrm{~nm}$ by Normalization I; (C') and $\left(E^{\prime}\right)$, normalized at 433 and $500 \mathrm{~nm}$ by Normalization II. The lowest solid line in (I) is the experimental baseline spectrum taken with no excitation. 
intensities, respectively. The absorption spectra in the range of 400$460 \mathrm{~nm}$ (with $\lambda_{\max }$ at 433 and $407 \mathrm{~nm}$ ) are similar to the $T_{n} \leftarrow T_{1}$ absorptions at longer times ${ }^{9-12}$ in regard to the positions of the absorption bands and the intensity distribution. The weak absorption above $450 \mathrm{~nm}$ in Spectrum C obtained by Normalization I does not appear in Spectrum $C^{\prime}$ obtained by Normalization II. A similar result was obtained by normalization of the weak absorption measured by reducing the sample concentration to one-tenth of the original one.

Apart from this weak absorption above $450 \mathrm{~nm}$, the normalized spectra at -10 ps (Spectra $E$ and $E^{\prime}$ in Figures 5-III and IV) evidently show broadening and shifts to a longer wavelength in comparison with that at 320 ps. Since no essential spectral narrowing and shifts are observed in Spectra $C$ and $C^{\prime}$ within experimental error, changes in the time-dependent spectral shape below $450 \mathrm{~nm}$ might be due to vibrationally unrelaxed acridine, as suggested for benzophenone by Hochstrasser et al. ${ }^{1}$

The weak absorptions of acridine observed above $450 \mathrm{~nm}$ in Spectra $\mathrm{C}$ and $\mathrm{E}$ are very similar to that observed in the first picosecond region, i.e., at 14 ps by Hochstrasser et al., who have assigned this spectrum to that of a transition between $n \pi^{*}$ configurations in the singlet states. However, we suspect that this weak absorption is a spurious one as a result of normalization analogous to Normalization I. This is based on the following reasons: (1) No such weak absorptions are observed by Normalization II. (2) From the normalized spectrum at 14 ps reported by Hochstrasser et al., one can estimate the ratio of the absorbance at $520 \mathrm{~nm}$ to that at $433 \mathrm{~nm}$ to be 0.48 . By contrast, our Spectrum $E$ at $-10 \mathrm{ps}$ indicates that the ratio is 0.15 , which is nearly equal to those estimated from published spectra ${ }^{1,9,13}$ measured at longer times, though the value 0.15 is smaller than the error arising from Normalization I, i.e., 0.23 in Spectrum C. (3) If these authors' suggestion is acceptable, one should observe an absorption of much higher intensity above $450 \mathrm{~nm}$, because we have clearly observed the absorption peak at $407 \mathrm{~nm}$ whose relative intensity to that at $433 \mathrm{~nm}$ is $0.46-0.58$.

As we have demonstrated in the present paper, time-dependent spectral changes in the first picosecond region sometimes depend on the procedure of spectral normalization. In spite of these circumstances, the spectral changes which might be due to vibrationally unrelaxed triplet states were observed in 9-acetylanthracene and 
acridine, whereas no spectral change was observed in benzophenone. No strong evidence has been provided which supports the results of Hochstrasser et al. in regard to the presence of $S_{1}$ absorption of acridine at 14 ps.

\section{Acknowledgement}

This research was financed by a grant-in-Aid for Scientific Research from the Japanese Ministry of Education, Science and Culture (No. 470006).

\section{References}

1. B. I. Greene, R. M. Hochstrasser and R. B. Weisman, J. Chem. Phys. 70, 1247 (1979).

2. E. C. Hawkins, J. Chem. Soc. 1957, 3858.

3. K. Hamanoue, T. Hidaka, T. Nakayama and H. Teranishi, Chem. Phys. Lett. 82, 55 (1981).

4. The absorption spectrum shifts to the red in benzene, ${ }^{5}$ giving two absorption maxima at 410 and $430 \mathrm{~nm}$. These absorptions are identical to the $T_{n} \leftarrow T_{1}$ absorption which has been observed by conventional flash photolysis at $77 \mathrm{~K}^{6}{ }^{6}$

5. T. Nakayama, S. Tai, K. Hamanoue and H. Teranishi, Mem. Fac. Ind. Arts, Kyoto Tech. Univ. 29, 46 (1980).

6. M. V. Alfimov, N. Ya. Buben, V. L. Glagalev, E. S. Kuyumdzhi, Yu. V. Pomazan and V. N. Shamshev, Opt. Spectry. 42, 267 (1977).

7. D. S. McClure and P. L. Hanst, J. Chem. Phys. 23, 1772 (1955).

8. T. Tsubomura, N. Yamamoto and S. Tanaka, Chem. Phys. Lett. 1, 309 (1967).

9. A. Kellmann and L. Lindqvist, ed. A. B. Zahlan, The Triplet State (Cambridge Univ. Press, London, 1967), p. 439.

10. E. J. Land, Proc. Roy. Soc. London A 305, 457 (1968).

11. Y. Hirata and I. Tanaka, Chem. Phys. Lett. 41, 336 (1976).

12. V. Sundstrom, P. M. Rentzepis and E. C. Lim, J. Chem. Phys. 66, 4287 (1977).

13. A. Kira, S. Kato and M. Koizumi, Bull. Chem. Soc. Japan 39, 1221 (1966). 